\title{
Neuropsychological syndrome in a patient with episodic howling and violent motor behaviour
}

\author{
G A Y LE G. MAR S H \\ From the Departments of Psychiatry and Neurology, Reed Neurological Research Center, \\ UCLA Center for the Health Sciences, Los Angeles, California, USA
}

SUMMARY A patient with atypical temporal lobe seizures involving violent behaviour was found to exhibit the neuropsychological syndrome described by Luria $(1966,1976)$ as pathognomonic of lesions in the depths of the left temporal lobe. This syndrome, mainly an audioverbal memory defect with relatively intact phonemic hearing, was evident in spite of an intact intellect.

It is often difficult to distinguish organically related seizures from simulated ones, particularly when the seizure involves unprovoked violent behaviour. A very small percentage of patients with temporal lobe epilepsy display episodic unprovoked rage and aggressive behaviour that appear to fit this syndrome related to dysfunction of the limbic system (Treffert, 1964; Lishman, 1968; Sweet et al., 1969; Mark and Ervin, 1970; Valenstein, 1973; Blumer, 1975; Walker and Blumer, 1975). Clinical neurological and psychiatric tests for assessing limbic system function, exclusive of invasive procedures, have been ineffective (Mark and Sweet, 1974). A relatively neglected approach is to assess psychological functions that have been thought to be mediated by the limbic system. Two limbic structures (hippocampus and amygdala) located in the mesiobasal temporal areas of the brain have been implicated in the mediation of memory, emotional states, autonomic, and somatic functions, and behaviour associated with the fight-orflight response (Slater et al., 1963; Malamud, 1967; Mark and Sweet, 1974; Wallack, 1976). Of these functions, memory appears to be the most objectively defined and amenable to measurement.

Luria (1966, 1973, 1976), Luria and Karasseva (1968), and Luria et al. (1967) have described a syndrome of impaired mental functioning in patients with lesions in the left midtemporal cortex or in the depths of the left temporal lobe. According to them, patients with lesions in these

Address for reprint requests: Dr Gayle G. Marsh, Neuropsychiatric Institute, The Center for Health Sciences, 760 Westwood Plaza, Los Angeles, California 90024, USA.

Accepted 21 November 1977 areas that disturb the relationship between the hippocampus and the left midtemporal cortex characteristically exhibit a modality-specific memory disorder with impaired retention of verbal material presented acoustically in spite of having relatively intact phonemic hearing. These patients can usually understand verbal material presented acoustically, can repeat individual sounds, words, and even short sentences, but when the amount of verbal material is increased to include a memory factor, they register only part of the material and confuse the order in which it is presented. Although they retain the general meaning of the material, they tend to substitute verbal and literal paraphasias when they reproduce the material. These patients demonstrate no appreciable degree of alienation of word meaning, often show no writing disorders, have intact reading and speech comprehension, and maintain a stable, active intention to memorise. Visual and auditory perception, visuospatial analysis and synthesis, and memory for verbal materials presented visually remain preserved. Memory for kinaesthetic, motor, and visual traces not easily verbally-coded also remains unaffected.

Although published reports illustrating this syndrome of impaired psychological functioning have been confined to patients with demonstrable lesions, the present case study extends the applicability of this syndrome of disturbed psychological functions to a patient in whom there is no radiological evidence of a visible lesion, but who demonstrates limbic system dysfunction by inference-for example, by an abnormal nasopharyngeal EEG recording. Luria's neuropsycho- 
logical tests were administered to a patient with a history of atypical violent outbursts.

\section{Case report}

The patient, a 29 year old right handed Caucasian male, had been confined in state mental hospitals for 19 years (from age 9 to 28 years) as an intractable violence-prone epileptic patient with psychosis. His prenatal and postnatal stages, and early childhood up to 13 months were reported normal when a severe viral infection, possibly meningitis or an encephalitis, was diagnosed; a second bout of viral infection occurred at age 7 years. Both illnesses resulted in hospitalisation for about 14 weeks. Sequelae from both illnesses included hyperactivity, irritability, temper tantrums, and unmotivated running. Violent behaviour, including assaults on others, began at age 9 years. Although epileptic activity was suspected, no well documented typical seizure activity had been observed over the years by hospital personnel other than his aggressive outbursts and irritability. $\mathrm{He}$ was admitted to UCLA Neurology Service at age 29 years for detailed study after becoming violently aggressive toward staff members in a nearby community emergency room.

Findings from neurological examination (exclusive of mental status), brain scan, and computerised tomograms were normal; surface EEG recording demonstrated diffuse slowing of background activity, and transient left and right mid to posterior temporal slow waves, but no focal epileptiform discharges were noted. $\mathrm{He}$ was alert and oriented; his speech was tangential and circumlocutory. Neither seizures nor aggressive attacks were noted on the ward until his anticonvulsant medications were decreased. A stereotyped seizure pattern of howling and running began 12 hours later and recurred repeatedly over the next few days, including days when placebo medication was administered. Occasionally, epigastric aura commonly associated with temporal lobe epilepsy preceded the attacks. At times he would exhibit automatisms of mouth movements or picking at his electrodes.

Nasopharyngeal EEG leads recorded frequent interictal spike and wave discharges along with sharp waves and slowing of activity in the left nasopharyngeal area. Videotaped monitoring during two clinical seizures demonstrated increased spike and wave discharges in the left nasopharyngeal region before and during the stereotyped aggressive motor behaviour, indicating that at least the initial stage of these episodes was an ictal event, involving the mesial area of the left tem- poral lobe. The uncinate area in the mesial temporal area is frequently the site of origin of electroencephalographic abnormality in temporal lobe epilepsy and has been implicated in the occurrence of aggressive attacks (Sweet et al., 1969; Mark and Sweet, 1974). Thus, the site of abnormal EEG activity in the present case appears to be important in the generation of aggressive motor behaviour and assaults. Anticonvulsant medication, carbamazepine $400 \mathrm{mg}$ twice daily, was substituted for the placebo without the patient's knowledge, and aggressive outbursts ceased, and he became calm and tractable. Six weeks later, the patient and his mother reported significant improvement in his behaviour, and a cessation of violent attacks.

\section{NEUROPSYCHOLOGICAL TEST FINDINGS}

Intelligence test

The patient, with seven years of formal schooling, obtained a Full Scale IQ (WAIS administered according to Satz and Mogel (1962), abbreviated form) of 98 (average range), placing him at the 45th percentile of his age group; verbal IQ of 83 (dull normal) and at the 12 th percentile; performance IQ of 118 (upper range of bright normal) and at the 90th percentile of his age group.

\section{Neuropsychological syndrome}

The patient revealed neuropsychological test signs characterised by Luria and Karasseva (1968) as pathognomonic of lesions in the middle segments of the left temporal cortex or located deep within the white matter of the left temporal cortex and destroying the hippocampus, mainly an audioverbal memory defect with relatively intact phonemic hearing. The patient could perceive immediately (or after a delay with no interference) single consonants, vowels, words, or short series of words. His recall became impaired when the range of words was expanded and when interference conditions were imposed during the delay interval by asking the patient extraneous questions. The patient could not retain the order of a series of words presented acoustically, and often could not give a complete recall of acoustic verbal materials. Perseverations of previous words, intrusions of extraneous verbal material, and substitution of literal and verbal paraphasias emerged under conditions of interference. His memory was particularly disturbed in recalling anecdotes. Although he was able to grasp the basic theme and retain it after a long period of time, he replaced the original text with morphologically or semantically related words so that paraphasias distorted the meaning of the anecdote. As expected, inter- 
ference conditions greatly increased his defective recall. Like Luria's patients with lesions of the deep regions of the left temporal lobe, recall of visual materials, including a story read silently by the patient, was retained better than verbal materials presented acoustically. $\mathrm{He}$ was able to retain new motor sequences learned during the neuropsychological investigation. His intellect remained relatively intact, considering his limited educational and social history.

\section{Discussion}

Surprisingly, in spite of the dissimilarity between the patients of Luria and Karasseva (1968) and the present case in respect to type of neurological disorder, presence of an actual lesion that could be visualised, presumed age of onset of the neurological disorder, and adequacy of educational and socialisation experiences, Luria's neuropsychological syndrome of an audioverbal memory disorder was found in the patient reported on here. The area of disturbed electrophysiological activity in the present case brings to mind the operated temporal lobe patients reported by Milner (1966). Her patients underwent a standard temporal lobe resection that ablated the anterior $60 \mathrm{~mm}$ of the temporal lobe including the hippocampus, hippocampal gyrus, uncus, and amygdala (Milner, 1966) - that area of the brain that showed abnormal epileptiform EEG activity in the present case. Milner reports that patients with left temporal lobe ablation exhibit a verbal memory defect that interferes with both the learning and retention of verbal material similar to the one reported here, except that it includes defective recall of verbal materials presented visually as well as acoustically. The probability that the patients operated on for temporal lobe epilepsy had more extensive loss of brain tissue in the hippocampal region due to surgical ablation than the present patient, who exhibited only intermittent abnormal EEG activity, may account for their more extensive memory disorder. In addition, in the present case, the patient suffered cerebral damage when his brain was relatively immature and plastic, and, therefore, more amenable to functional reorganisation. However, this difference may be more apparent than real. Although the present patient's registration and delayed recall of verbal materials presented visually were more intact than that presented acoustically, his performance was inferior to that of a neurologically intact individual. A reliable relative gave corroborative, anecdotal information that the patient has marked difficulty in retaining written material, even though he is highly motivated to learn.

The patient's difficulty in retaining the sequence of a series of verbal materials presented acoustically, the marked reduction in the amount of audioverbal material he was capable of registering and retaining, and the intrusions of audioverbal perseverations suggest that the underlying causal factor is one of increased inhibition of audioverbal traces as suggested by Luria (1976, page 179). Both proactive and retroactive inhibition appeared to be interfering with his memory for audioverbal stimuli.

\section{Conclusion}

In the present case, Luria's neuropsychological experimental tests were found to be effective in uncovering an audioverbal memory disorder in an epileptic patient with no demonstrable brain lesion that could be visualised by radiological techniques but who demonstrated abnormal epileptiform EEG activity in the mesial temporal area of the dominant (left) hemisphere. Neuropsychological test signs characterised by Luria and Karasseva (1968) as pathognomonic of lesions in this area include a modality-specific acoustic memory disorder involving verbal materials, whereas memory for visually presented verbal materials, kinaesthetic traces, and motor responses are better retained.

Luria's explanatory concepts of retroactive and proactive inhibition for the observed audioverbal memory disorder appear to be the most parsimonious hypothesis to explain the observed memory deficit.

I wish to thank Dr Gregory Walsh for his suggestions and interpretation of the EEG records.

\section{References}

Blumer, D. (1975). Temporal lobe epilepsy and its psychiatric significance. In Psychiatric A spects of Neurologic Disease, pp. 171-198. Edited by D. F. Benson and D. Blumer. Grune and Stratton: New York.

Lishman, W. A. (1968). Brain damage in relation to psychiatric disability after head injury. British Journal of Psychiatry, 114, 373-410.

Luria, A. R. (1966). Higher Cortical Functions in Man. Basic Books: New York.

Luria, A. R. (1973). The Working Brain: An Introduction to Neuropsychology. Penguin Press: London.

Luria, A. R. (1976). The Neuropsychology of Memory. John Wiley and Sons: New York.

Luria, A. R. and Karasseva, T. A. (1968). Disturbances of auditory-speech memory in focal lesions of 
the deep regions of the left temporal lobe. Neuropsychologia, 6, 97-104.

Luria, A. R., Sokolov, E. N., and Klimkowski, M. (1967). Towards a neurodynamic analysis of memory disturbances with lesions of the left temporal lobe. Neuropsychologia, 5, 1-11.

Malamud, N. (1967). Psychiatric disorder with intracranial tumors of limbic system. Archives of Neurology (Chicago), 17, 113-123.

Mark, V. H., and Ervin. F. R. (1970). Violence and the Brain. Harper and Row: New York.

Mark, V. H., and Sweet, W. H. (1974). The role of limbic brain dysfunction in aggression. In Aggression, pp. 186-198. Research Publications. Association for Research in the Nervous and Mental Diseases, 52. Williams and Wilkins Co.: Baltimore.

Milner, B. (1966). Amnesia following operation on the temporal lobes. In Amnesia, pp. 109-133. Edited by C. W. M. Whitty and O. L. Zangwill. Butterworths: London.

Satz, P., and Mogel, S. (1962). An abbreviation of the WAIS for clinical use. Journal of Clinical Psychology, 18, 77-79.
Slater, E., Beard, A. W., and Glithero, E. (1963). The schizophrenia-like psychoses of epilepsy. British Journal of Psychiatry, 109, 95-150.

Sweet, W. H., Ervin, F., and Mark, V. H. (1969). The relationship of violent behaviour to focal cerebral disease. In Aggressive Behavior, pp. 336-352. Edited by S. Garattini and E. G. Sigg. Proceedings of the International Symposium on the Biology of Aggressive Behavior. Milan, 2-4 May 1968. John Wiley and Sons: New York.

Treffert, D. A. (1964). The psychiatric patient with an EEG temporal lobe focus. American Journal of Psychiatry, 120, 765-771.

Valenstein, E. S. (1973). Brain Control: A Critical Examination of Brain Stimulation and Psychosurgery. John Wiley and Sons: New York.

Walker, E. A., and Blumer, D. (1975). Long-term effects of temporal lobe lesions on sexual behavior and aggressivity. In Neural Bases of Violence and Behavior, pp. 392-400. Edited by W. S. Fields and W. H. Sweet. Warren H. Green, Inc.: St Louis.

Wallack, E. (1976). Selective deficits after encephalitis. Southern Medical Journal, 69, 669-672. 